the deep muscles in the side of the neck, and moving the head freely in all directions.

$$
1 \text { St. Bartholomew's Hospital Journal. }
$$

DIET IN TYPHOID FEVER.

AT a meeting of the South-Western Branch of the British Medical Association Dr. Norway drew attention afresh to a point which constantly crops up when the subject of typhoid fever is discussed by practical men, namely, the importance of a dietary which shall not leave the intestines charged with undigested and decomposing débris, and the inappropriateness of milk as ordinarily given as a food for such cases. Dr. Norway says that in several necropsies which he made and witnessed on typhoid patients in a London hospital he was struck by the undigested condition of the food found in the stomach and intestines. He has removed from a stomach a mass as large as, and resembling a Spanish onion, which consisted of laminated curdled milk ; and in no case did the food found in the stomach or intestines appear to be digested. The food was much as it was before it was swallowed, except that the milk was curdled and the contents of the ileum were very offensive. With these facts before him, together with other theoretical considerations which need not now detain us, Dr. Norway has treated his patients with a mixture by aid of which he hopes to enable them to digest the nourishment given. For an adult the following formula is employed. R Essen. Pepsintiæ (Armour), fl. dr. $\frac{1}{2}$ to fl. dr. i ; acid nitro-hydro-chlorici dil., $m x$; glycerini, $\operatorname{mxx}$; aquam. ad. fl. oz., $\frac{1}{2}$. This half ounce of solution is to be placed in half a tumbler, or more, of water, and to be sipped or taken as desired to relieve thirst, such a dose being at first given every hour, but as the temperature falls the frequency of the dose being gradually lessened. The mixture, however, is to be given throughout the illness until pain in the iliac fossa disappears and the stools become normal. The diet consists as far as possible of milk (unboiled, but from a carefully selected dairy). To vary the flavour tea or coffee may be added. Five or six ounces of milk may be given every two hours. Occasional meals of some form of beef tea should be given to prevent the patient becoming too tired of milk, and very soon bread and milk, egg and milk, arrowroot, milk puddings, and scrambled eggs may be given, but a daily inspection of the stools can alone decide a change of diet.

\section{THE TREATMENT OF TUBERCULOUS}

$$
\text { PERITONITIS. }
$$

Is a lecture on Tuberculous Peritonitis delivered at Guy's Hospital, Dr. Hale White ${ }^{1}$ recently gave some useful hints as to the choice of case for operation. In regard to the operation itself all authorities are agreed that laparotomy is much more reliable than puncture. Some surgeons, he says, wash out the abdomen with boiled water, others use weak anti. septics, others dust iodoform on some parts, others excise caseous nodules, and some take away the Fallopian tubes if the genital organs are tuberculous; "but as all surgeons appear to get equally good results, it seems clear that the chief point is merely opening the abdomen." As to whether to operate or not in any given case he points out that one should never operate if the peritonitis is merely a part of an acute general tuberculosis. Next, operation must not be done if there is already a fæcal fistula, for the presence of this condition shows that there must be numerous adhesions, and that one will in all probability not be able to get into the peritoreal cavity at all. It must be remembered that the best results are obtained in cases in which there is most fluid present. These are the very cases which do best whether operated on or not. Still, they are just the cases in which the risk of operation is exceedingly small, so that it is probably wise to open the abdomen and let the fluid out, especially if it be impossible to send the patient away into the fresh air. The best thing to do would probably be to open the abdomen, let out the fluid, and then send the patient away into the country. The worst results are obtained when there is little or no fluid in the abdomen; in these cases, therefore, the operation should not be done. Localised collections should be dealt with on the same principles. Localisation, however, points to the presence of adhesions, so one should be chary of touching them. The prominent umbilicus that is so often met with in these cases must never be incised. It often means that there is a tuberculous abscess under it which will sooner or later open into the bowel, and if it has been previously opened externally one will then have a fæcal fistula to deal with. Finally, if there should be a fæcal fistula one must not on any account try to deal with it by operation. The most disastrous results sometimes follow, and Dr. Hale White has never seen any good done by such attempts.

1 Guy's Hospital Gazette, June 21.

\section{PINEAPPLE JUICE IN MEDICINE.}

A GOOD deal has lately been written about the digestive action of fresh pineapple. It has been pointed out that a freshly-cut slice of pineapple laid on a piece of beef steak will in a comparatively short time cause softening, swelling, and partial digestion of the meat for a considerable depth from the surface. It is also stated that bromoline the active principle of the pineapple has long been used in the preparation of the well-known Masquera's beef jelly. Dr. Wyatt Wingrave ${ }^{1}$ says that the reputation of the pine has suffered, among other reasons, from the facts that far too much is eaten at a time, and that the fibrous part is swallowed as well as the juice. To obtain its full digestive value one quadrant of a slice half an inch thick is ample for one meal. It should be well masticated and the fibrous portion should be rejected. It must not be cooked, and should be just ripe. The preserved form has practically no digestive power. Apart from its use as a digestive, the juice has a strong solvent action upon plastic exudation, such as diphtheria membrane. When applied to such a membrane on a swab, or as a spray, its time of contact is not enough to cause solution, but it is of material service in softening the sticky and stringy exudation so as to admit of its easy detachment. It also softens horny epidermis in the same way as, although more slowly than, salicylic acid. If a thin slice of pineapple be kept in close contact with a corn for eight hours it will be so softened as to admit of ready removal. Again, it softens the horny papillæ in keratosis of the tonsil, and quickly relieves the prickly sensation complained of in that condition. 